\title{
THE PLECOPTERA AND TRICHOPTERA OF THE ARCTIC NORTH SLOPE OF ALASKA
}

\author{
Michael R. Kendrick ${ }^{1,2}$ and Alexander D. Huryn ${ }^{1}$
}

\begin{abstract}
The Arctic is currently experiencing changes in climate more rapid than in any other biome. This warming trend has resulted in significant abiotic changes to the seasonal patterns of freshwater ecosystems. Thorough inventories of freshwater insect communities are required to provide benchmarks for the detection of range shifts in response to a warming climate. Though statewide studies have been conducted for Trichoptera and Plecoptera, species accounts for these orders in Arctic Alaska have received relatively little attention. We surveyed Plecoptera and Trichoptera of Alaska's Arctic North Slope at a variety of habitat types over an 11-year period. We document new and historical collection records for 24 species of Plecoptera and 33 species of Trichoptera on Alaska's North Slope. Among these are 19 new North Slope records for Trichoptera (4 new state records) and 2 new North Slope records for Plecoptera. Our assessment of these taxa reveals communities of stoneflies and caddisflies that are distinct to Alaska's North Slope and should be of clear conservation concern. Two major factors contributing to these unique communities include the function of the North Slope as a Beringian refuge from continental ice sheets and the occurrence of spring streams as refuge from seasonal winter ice. The North Slope of Alaska is predicted to undergo changes in climate and industrialization in coming years, and this species list will aid our understanding of how aquatic insect communities may respond to future changes in Arctic Alaska.
\end{abstract}

Resumen.-El Ártico atraviesa actualmente cambios climáticos más rápidos que cualquier otro bioma. Esta tendencia al calentamiento ha resultado en cambios estacionales abióticos significativos en los patrones de los ecosistemas de agua dulce. Se requieren minuciosos inventarios de comunidades de insectos de agua dulce para proporcionar puntos de referencia que permitan la detección de cambios en el nivel de respuesta a un calentamiento climático. Si bien se han conducido estudios de Trichoptera y Plecoptera en todo el estado, recuentos de las especies de estos órdenes en el Ártico en Alaska han recibido relativamente poca atención. Examinamos la plecópteros y tricópteros de la ladera norte del Ártico en Alaska en una variedad de hábitats durante un período de 11 años. Documentamos nuevos e históricos registros de colecciones de 24 especies de Plecoptera y 33 especies de Trichoptera en la ladera norte de Alaska. Entre ellos se encuentran 19 nuevos registros de Trichoptera de la ladera norte (4 nuevos registros de estado) y 2 nuevos registros de Plecoptera en la ladera norte. Nuestra evaluación de estos taxa revela comunidades de insectos del orden Plecoptera distintos de los de la ladera norte de Alaska y deberían evidentemente ser de preocupación de conservación. Dos factores principales que contribuyen a estas comunidades únicas incluyen la ladera norte como un refugio Beringiano de las placas de hielo continental y la existencia de corrientes primaverales como refugio de la estación de hielo invernal. Se prevé que la ladera norte de Alaska sufrirá cambios en el clima e industrialización en años venideros y esta lista de especies ayudará a nuestro entendimiento de cómo las comunidades de insectos acuáticos pueden responder a futuros cambios en el Ártico de Alaska.

Climate change is currently altering ecosystem structure and function in Arctic ecosystems, where temperatures are rising due to the polar amplification of greenhouse warming (Hinzman et al. 2005, Chapin et al. 2006, Martin et al. 2009). This warming trend has already resulted in significant changes in the seasonality of freshwater habitats in Arctic and Boreal North America, where the spring breakup of winter river ice has showed successively earlier dates over the past 6 decades (White et al. 2007, de Rham et al. 2008, Tape et al. 2011). Increasing temperatures are highly correlated with the range extensions of many insect species (Chen et al. 2011), and it is thus likely that populations of freshwater insects in the Arctic are undergoing range adjustments in response to climate warming (ACIA 2005). Similarly, it is anticipated that lower-latitude species will eventually expand their ranges into the Arctic (ACIA 2005). Thorough inventories of freshwater insect communities are thus required to provide benchmarks for the detection of range shifts of species in response to a warming climate. Unfortunately, records documenting the

\footnotetext{
${ }^{1}$ Department of Biological Sciences, University of Alabama, Tuscaloosa, AL 35487-0206.

${ }^{2}$ E-mail: kendrickmr@gmail.com
} 
distributions of freshwater insect species of the Arctic regions of Alaska are limited. The distribution of the Ephemeroptera (McCafferty 1985, Randolph and McCafferty 2005), Plecoptera (Stewart and Oswood 2006), and Trichoptera (Nimmo 1986) in Alaska have been documented at the statewide level. Few studies, however, have focused exclusively on the Arctic. Weber (1950), for example, is the only relatively comprehensive source that includes records for the occurrence of freshwater insect taxa while specifically treating the North Slope, where changes in the ranges of Alaskan insects as a result of climate warming are anticipated to be most significant.

Herein we provide an updated synopsis of new and historical distribution records of the Plecoptera and Trichoptera of the Arctic North Slope (including the Noatak River basin) of Alaska. It is our intent that this synopsis will provide a readily available benchmark for the detection of future changes in the ranges of freshwater invertebrates in this region.

\section{Methods}

We conducted this study on the central and eastern North Slope of Arctic Alaska. The North Slope is a relatively large region with an area roughly equal to that of Nebraska $\left(\sim 200,000 \mathrm{~km}^{2}\right)$ and is bounded on the north by the Arctic Ocean and on the south by the Brooks Range. It is subject to average monthly air temperatures of -30 to $-27^{\circ} \mathrm{C}$ in January (warmer temperatures near the coast of the Arctic Ocean; colder temperatures inland) and 5 to $13{ }^{\circ} \mathrm{C}$ in July (colder temperatures near the coast; warmer temperatures inland). The North Slope typically receives a minimum of $150 \mathrm{~mm}$ of annual precipitation near the coast and $550 \mathrm{~mm}$ of precipitation in some areas of the Brooks Range; snowfall is possible on any day of the year (Huryn and Hobbie 2012). This treeless tundra region is underlain by a continuous layer of permafrost and is covered by vegetation dominated by cottongrass (Eriophorum), dwarf birches (Betula), willows (Salix), mosses (primarily Sphagnum), sedges (Carex), and lichens (Huryn and Hobbie 2012). Stoneflies (Plecoptera) and caddisflies (Trichoptera) were collected during the summer periods of 2001-2012. Adults were collected by hand and aerial net along rivers, streams, springs, wetlands, ponds, and lakes in habitats ranging from alpine to coastal plain from locations primarily in the Kuparuk, Sagavanirktok, Ivishak and Noatak River drainages of the central and eastern North Slope (Table 1). Larvae were collected in the same habitats and locations by use of kicknets and other sampling devices. Light traps, which are extremely effective for collecting adult caddisflies, could not be used due to continuous daylight during summer. As a consequence, sticky traps $(n=12)$ were deployed in the riparian zones of 8 streams along the North Slope section of the Dalton Highway (30 June-9 August 2011) and recovered at $\sim 1$-week intervals. These traps consisted of standard overhead projector transparencies that were coated with Tanglefoot ${ }^{\circledR}$ (The Tanglefoot Company, Grand Rapids, MI). Voucher specimens from this collection will be available through Brigham Young University's insect collection (R. Baumann personal communication).

\section{Species LisT}

New and historical records documenting the occurrence of Plecoptera and Trichoptera are provided in the following list. The familylevel structure of the list follows Stewart and Oswood (2006) for the Plecoptera and Wiggins (1996) for the Trichoptera. Records taken from the literature, and not from our 2001-2012 collections, are indicated by the following superscripts:

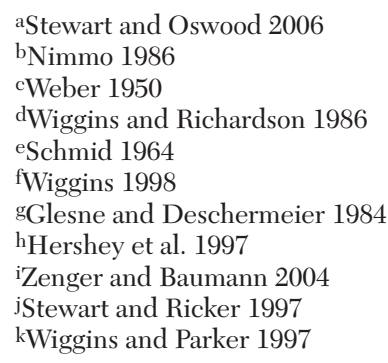

Previously undocumented Alaskan North Slope records are preceded by an asterisk (*), while new Alaska state records are preceded by 2 asterisks (**). Date format is $\mathrm{dd} / \mathrm{mm} / \mathrm{yyyy}$, and specimens are characterized as " $\mathrm{m}$ " for males and "f" for females. Names of species documented from the Arctic north slope drainages of the Yukon Territory, Canada are preceded by a double dagger ( $\ddagger)$ and are included for reference, but only Alaska specimens are included in further analyses. 
TABLE 1. Location of collection sites inventoried from 2001 to 2012. Sites where sticky traps were deployed are indicated by an asterisk (*).

\begin{tabular}{|c|c|c|}
\hline Site name & Latitude & Longitude \\
\hline Barrow Environmental Observatory & $71.277075^{\circ}$ & $-156.639441^{\circ}$ \\
\hline Cobblestone River aufeis ${ }^{\mathrm{a}}$ & $68.6404^{\circ}$ & $-150.59554^{\circ}$ \\
\hline Dan Creek* & $69.098067^{\circ}$ & $-148.8331^{\circ}$ \\
\hline Echooka Spring & $69.266944^{\circ}$ & $-147.203056^{\circ}$ \\
\hline Franklin Bluffs near Dalton Highway Milepost 386 & $69.785596^{\circ}$ & $-148.654633^{\circ}$ \\
\hline Galbraith Lake & $68.465235^{\circ}$ & $-149.434191^{\circ}$ \\
\hline Galbraith Lake "west" aufeis & $68.454833^{\circ}$ & $-149.455083^{\circ}$ \\
\hline Galbraith Lake "campground spring" & $68.4529^{\circ}$ & $-149.480367^{\circ}$ \\
\hline Happy Valley Creek* & $69.15065^{\circ}$ & $-148.835333^{\circ}$ \\
\hline Hershey Creek* & $68.644399^{\circ}$ & $-149.412706^{\circ}$ \\
\hline Holden Creek & $68.406117^{\circ}$ & $-149.325333^{\circ}$ \\
\hline Imnavait Creek & $68.619069^{\circ}$ & $-149.318544^{\circ}$ \\
\hline Ivishak Hot Spring & $69.029444^{\circ}$ & $-147.664167^{\circ}$ \\
\hline Krugurak Spring & $67.623917^{\circ}$ & $-155.622533^{\circ}$ \\
\hline Kuparuk River* (Dalton Highway bridge) & $68.638933^{\circ}$ & $-149.393067^{\circ}$ \\
\hline Kuparuk River "lower" aufeis & $68.988017^{\circ}$ & $-149.731433^{\circ}$ \\
\hline Kuparuk River "upper" aufeis & $68.59295^{\circ}$ & $-149.357867^{\circ}$ \\
\hline Lake E-1 outlet* near Toolik Lake & $68.6359^{\circ}$ & $-149.584717^{\circ}$ \\
\hline Lake E5 near Toolik Lake & $68.645067^{\circ}$ & $-149.460183^{\circ}$ \\
\hline Lake I-3 outlet stream near Toolik Lake & $68.575233^{\circ}$ & $-149.582915^{\circ}$ \\
\hline Lake I-6 headwater outlet stream near Toolik Lake & $68.584031^{\circ}$ & $-149.62409^{\circ}$ \\
\hline Lupine Creek* & $69.305983^{\circ}$ & $-148.741967^{\circ}$ \\
\hline May Creek spring and aufeis & $68.682778^{\circ}$ & $-150.418611^{\circ}$ \\
\hline Mosquito Lake & $68.447303^{\circ}$ & $-149.367817^{\circ}$ \\
\hline Oil Spill Hill, Dalton Highway & $68.942975^{\circ}$ & $-148.86843^{\circ}$ \\
\hline Oksrukuyik River* & $68.687217^{\circ}$ & $-149.09535^{\circ}$ \\
\hline Percy Pingo & $70.005645^{\circ}$ & $-148.76337^{\circ}$ \\
\hline Ribdon River spring & $68.473889^{\circ}$ & $-148.149444^{\circ}$ \\
\hline Roche Moutonnee Creek & $68.372773^{\circ}$ & $-149.31905^{\circ}$ \\
\hline Sagavanirktok River "glacier tributary" & $68.361111^{\circ}$ & $-149.077222^{\circ}$ \\
\hline Sadlerochit Spring & $69.664722^{\circ}$ & $-144.414444^{\circ}$ \\
\hline Tea Lake & $68.423106^{\circ}$ & $-149.375748^{\circ}$ \\
\hline Toolik Lake & $68.63115^{\circ}$ & $-149.608117^{\circ}$ \\
\hline Toolik River near Dalton Highway & $68.647283^{\circ}$ & $-149.319217^{\circ}$ \\
\hline Toolik River aufeis & $68.121283^{\circ}$ & $-149.320083^{\circ}$ \\
\hline Wolf Creek* & $68.670517^{\circ}$ & $-149.127583^{\circ}$ \\
\hline
\end{tabular}

${ }^{\mathrm{a} A u f e i s ~ a r e ~ s p r i n g-a s s o c i a t e d ~ i c e ~ f i e l d s ~(s e e ~ d i s c u s s i o n) . ~}$

\section{PLECOPTERA}

\section{Capniidae}

\section{Capnia confusa Claassen, 1936}

New RECORDS.—Toolik Lake, If, 28/6/2001; Ivishak Hot Spring stream, $1 \mathrm{~m} \&$ 2f, 15/7/2002, $1 \mathrm{~m}$ \& 4f, 23-28/6/2002; Ivishak River, main stem near Ivishak Hot Spring, 2m, 28/5/2008; Cobblestone River aufeis, 1m, 8/6/2012; May Creek aufeis, $1 \mathrm{~m} \&$ 1f, 8/6/2012.

Historical RECORDS.-Anaktuvuk River, 1949a,c; Shublik Spring, 1966a; Echooka Spring, 1971ª, Canning River, unnamed spring, 1972 ${ }^{\mathrm{a}}$.

\section{Capnia nearctica Banks, 1918}

NEW RECORDS.-Toolik Lake, $1 \mathrm{~m} \& 6 \mathrm{f}$, 29/6/2001, 1f, 21/7/2001; May Creek spring, lf, 8/7/2001; Toolik River aufeis, 1f, 11/7/2001; Ku- paruk River “upper” aufeis, 2m \& 7f, 29/6/2007; Ivishak River near Ivishak Hot Spring, 4f, 28/5/2008; Ivishak River, main stem near Ivishak Hot Spring, 8m, 28/5/2008; Galbraith Lake "west" aufeis, 1 brachypterous $1 \mathrm{~m} \& 3 \mathrm{f}$, 3/6/2012; Kuparuk River "lower" aufeis, $1 \mathrm{~m}$ \& 8f, 8/6/2012; May Creek spring, lf, 1/6/2012, $5 \mathrm{~m}, 8 / 6 / 2012$; Toolik River aufeis, 1 brachypterous male, 8/6/2012; Kuparuk River (Dalton Highway bridge), 1 brachypterous male, 22/6/ 2012; Kuparuk River "lower" aufeis, 1 brachypterous male, 10/8/2012.

HiSTORICAL RECORDS.-Umiat, 1948a; Anaktuvuk River, 1949ª,c; Toolik Lake, 1979a .

\section{†Capnia pileata Jewett, 1966}

Historical ReCORDs.-Philip Creek, Arctic slope of Yukon Territory, Canada (no date)j. 
Isocapnia abbreviata Frison, 1942

HistoriCAL RECORDS.-Noatak River, $1973^{\mathrm{i}}$.

Isocapnia crinita (Needham \& Claassen, 1925)

NEW RECORD.-Cobblestone River aufeis, 2f, 8/6/2012.

HistoriCAL RECORDS.-Noatak River, 1973i.

Isocapnia grandis (Banks, 1907)

NEW RECORDS.-Ribdon River spring, 2m, 1/7/2001; Cobblestone River aufeis, lf, 8/6/2012.

Historical RECORDS.-Canning River, spring tributary, $1972^{\mathrm{a}}$.

*Isocapnia cf. integra Hanson, 1943

New RECORDS.-Cobblestone River aufeis, lf, $8 / 6 / 2012$.

Mesocapnia oenone (Neave, 1929)

Historical ReCords.-Anaktuvuk Pass, $1949^{c}$.

Mesocapnia variabilis (Klapálek, 1920)

NEW RECORDS.-Echooka Spring, lf, 4/7/ 2001; Kuparuk River (Dalton Highway bridge), 3f, 20/7/2001, 2m \& 3f, 3/8/2001; Sadlerochit Spring, 2f, 22/7/2002; Kuparuk River (Dalton Highway bridge), 2f, 3/8/2012; Galbraith Lake "west" aufeis, lf, 22/8/2012; Kuparuk River "upper" aufeis, 5m, 10/8/2012; Toolik River aufeis, $2 \mathrm{~m} \& 5 \mathrm{f}, 10 / 8 / 2012$.

Historical RECORDS.-Anaktuvuk River, 1948; ; Cape Thompson, 1960ª Canning River, 1972a; Cache Creek, 1972a; Atigun Pass, 1980a; Atigun River, 1980ª Pump Station 4, Dalton Highway, 1980a; Roche Moutonnee Creek, 1980a; Sikrelurak River, 1982a.

Nemouridae

$\$$ Nemoura arctica Esben-Peterson, 1910

NEW RECORDS.-Sagavanirktok River "glacier tributary," $2 \mathrm{~m}$ \& 1f, 10/6/2001; Toolik Lake, $1 \mathrm{~m}, 26 / 6 / 2001,2 \mathrm{~m} \& 2$ f, 28/6/2001; Ribdon River spring, 1f, 1/7/2001; Echooka Spring, $1 \mathrm{~m} \&$ 2f, 4/7/2001; Kuparuk River (Dalton Highway bridge), $1 \mathrm{~m} \& \mathrm{lf}, 4 / 7 / 2001,1 \mathrm{~m} \& 1 \mathrm{f}$, 11/7/2001, lm, 20/7/2001, lf, 9/8/2001; Kuparuk River "upper" aufeis, $1 \mathrm{~m}$ \& 2f, 20/7/ 2001; Ivishak Hot Spring stream, If, 23-28/6/ 2002; Ivishak Hot Spring stream, 1f, 26/5/2008; Ivishak Hot Spring, hillslope source, 36m \& 25f, 26-29/5/2008; Kuparuk River (Dalton Highway bridge), 1f, 18/6/2012, 1m \& 6f, 19/6/
2012, $1 \mathrm{~m} \&$ \&f, 22/6/2012, $1 \mathrm{~m} \&$ \& 16f, 23/6/2012; Oksrukuyik Creek, lf, 5/7/2012; Lupine Creek, 2f, 9/7/2012; Holden Creek, 1f, 11/7/2012; Roche Moutonnee Creek, 1f, 11/7/2012.

Historical RECORDS.-Anaktuvuk Pass, 1948-1949c; Point Barrow, 1949a; Point Barrow, 1955a; Cape Lisburne, 1957a; Contact Creek, 1957a; Umiat, 1959a; Cape Thompson, $1960^{\mathrm{a}}, 1961^{\mathrm{a}}$; Cape Lisburne, 1965a ; Shublik Spring, 1969a; Anaktuvuk Pass, 1968a; Ribdon River (S. Fk.), 1971'a; Section Creek, 1971a; Echooka River, 1971ª, Echooka Spring, 1971a; Cane Creek, 1972a; Sadlerochit Spring, 1972a; Canning River tributaries, 1972a, 1973a; Kikitaliorak Lake, 1973a; Feniak Lake, 1973a; Schrader Lake, 1973a; Galbraith Lake, 1976a; Atigun River and tributaries, 1977a; Kuparuk River (Dalton Highway bridge), 1978a; Toolik Lake, 1979a; Atigun River and tributaries, 1980a; Pump Station 4, Dalton Highway, 1980a; Teshekpuk Lake, 1980a; Oksrukuyik Creek, 1980a; Roche Moutonnee Creek, 1980a; Okerokovic River, 1982a; Jago River, 1982a; Kaktovik, 1982a; Aichilik River, 1982a; Angun River, 1982a; Oksrukuyik Creek, 1982a; Storkerson Point, 1982a; Peters Lake, 1982a; Nuvvgapak Point, 1982a; VABM Mars, 1982a; Roche Moutonnee Creek, 1986a; Toolik River, no date $^{\text {a }}$ Philip Creek, Arctic slope of Yukon Territory, Canada (no date)j.

Nemoura normani (Ricker, 1952)

Historical RECORDS.-Anaktuvuk River, $1949^{\mathrm{a}}$.

Podmosta decepta (Frison, 1942)

HistoriCAL RECORDS.-Umiat, 1959a.

Podmosta weberi (Ricker, 1952)

NEW RECORDs._Kuparuk River "lower" aufeis, lm \& 1f, 11/6/2001, lf, 19/6/2004; Galbraith Lake "campground spring," $1 \mathrm{~m} \& 3 \mathrm{f}$, 24/6/2007, 10m \& 8f, 29/6/2007; Kuparuk River "lower" aufeis, $1 \mathrm{~m} \&$ 1f, 10/8/2012.

Historical RECORDS.-Anaktuvuk Pass, 1949ª Anaktuvuk River, 1949ª

\section{Soyedina sp.}

Historical RECORDS.-Kikitaliorak Lake, larvae, 1973a.

Zapada columbiana (Claassen, 1923)

Historical Records.-Shublik Spring, 1968, 1969; Echooka Spring, 1971ª; Ribdon 
River, spring tributary, 1971a; Canning River, 1972a; Shublik Spring, 1982a.

\section{Zapada haysi (Ricker, 1952)}

NEw RECORDS.-Echooka Spring, 1f, 4/7/ 2001, If, 18/7/2004; Ivishak Hot Spring stream, 2f, 9/7/2001, 3m \& 8f, 23-28/6/2002, 1m \& 1f, 30/7/2002 [Dolly Varden char (Salvelinus malma) gut contents]; Ivishak River tributary nr. Ivishak Hot Spring, 19m, 28/5/2008; Ivishak River nr. Ivishak Hot Spring, 10m \& 11f, 28/5/2008; Ivishak Hot Spring stream, 14m \& 6f, $26 / 5 / 2008$.

HistoriCAL RECORDS.-Anaktuvuk River 1949c; Shublik Spring, 1966ª, Lupine River, spring tributaries, 1971a; Echooka Spring, 1971a, 1972a; Canning River, Marsh Fork 1972a; Echooka River, 1972a; Shublik Spring, 1972a; Canning River, spring tributary, 1972a ${ }^{\mathrm{a}}$ 1973 ${ }^{\mathrm{a}}$.

\section{Taeniopterygidae}

\section{Taenionema pacificum (Banks, 1900)}

NEW RECORDS.-Ivishak Hot Spring stream, $2 \mathrm{~m} \& 2 \mathrm{f}, 23-28 / 6 / 2002$; Ivishak River, main stem nr. Ivishak Hot Spring, $19 \mathrm{~m} \& 23 \mathrm{f}$, 28/5/2008.

HistORICAL RECORDS.-Canning River, spring tributaries, 1973a, Ribdon River, South Fork, 1971'a.

\section{Chloroperlidae}

Alaskaperla ovibovis (Ricker, 1965)

NEW RECORDS.-Kuparuk River (Dalton Highway bridge), 18m \& 12f, 20/7/2001, 4m \& 4f, 3/8/2001; Lupine Creek, 1m, 9/7/2012.

HistoRiCAL RECORDS.-Kuparuk River, larvae, no date ${ }^{h}$.

\section{\$Plumiperla diversa (Frison, 1935)}

New RECORDS.-Echooka Spring, 3m, 4/7/ 2001; Ivishak Hot Spring stream, 2m, 9/7/2001, 1m, 6/8/2001; Kuparuk River "lower" aufeis, 2m, 11/7/2001; Ribdon River spring, 3f, 15/7/ 2001; Kuparuk River (Dalton Highway bridge), $1 \mathrm{~m} \& 2 \mathrm{f}, 11 / 7 / 2001,3 \mathrm{~m} \&$ \& 1 , 23-28/6/2002; Ivishak Hot Spring stream, 1m, 30/7/2002 (among S. malma gut contents); Sadlerochit Spring, 1m, 22/7/2002; Kuparuk River "lower" aufeis, 2f, 19/6/2004; Ivishak Hot Spring stream, 2m, 2-6/7/2007; Ivishak River tributary nr. Ivishak Hot Spring, 1m, 5/7/2007.
HistoriCAL RECORDS.-Anaktuvuk River, 1949a,c; Ribdon River, 1971a; Ribdon spring tributary, 1971; Lupine River, 1971a; Echooka River, 1971a; Echooka Spring, 1971a; Canning River, spring tributaries, 1972a, 1973a; Oksrukuyik Creek, 1980a; Shublik Spring, 1982a; Philip Creek, Arctic slope of Yukon Territory, Canada (no date)j.

\section{Suwallia autumna (Hoppe, 1938)}

Historical ReCORDS.-Sadlerochit Spring, $1974^{\mathrm{a}}$.

Suwallia starki Alexander \& Stewart 1999

NEW RECORDS.-Cobblestone River aufeis, $2 \mathrm{~m}, 8 / 6 / 2012$; Kuparuk River "lower" aufeis, $1 \mathrm{~m}, 10 / 8 / 2012$.

HistoriCAL RECORDS.-Anaktuvuk River, 1949a; Echooka Spring, 1971a; Umiat, 1959ª

*Paraperla frontalis (Banks, 1902)

NEW RECORDs.-Ribdon River spring, 3m, 1/7/2001, 1m, 15/7/2001.

$$
\text { Perlodidae }
$$

Isoperla decolorata (Walker, 1852)

Historical RECORDS.-Umiat, 1959a; Echooka Spring, 1971 ${ }^{\mathrm{a}}$.

\section{Isoperla petersoni Needham \& Christenson 1927}

New RECORDS.-Echooka Spring, $5 \mathrm{~m} \&$ 7f, 4/7/2001; Ivishak Hot Spring stream, 1m, 9/7/2001, lf, 6/8/2001, 1m, 15/7/2002, $2 \mathrm{~m} \& 2 \mathrm{f}$, 23-28/6/2002; Sadlerochit Spring, 9m, 22/7/ 2002; Ivishak Hot Spring stream, 1m, 26/5/ 2008; Ivishak Hot Spring, hillslope source, 2 larvae, 26-29/5/2008.

Historical RECORDS.-Shublik Spring and Creek, 1969a; Echooka River, 1971'a, Echooka Spring, 1971a; Ribdon spring tributaries, 1971a; Marsh Fork of Canning River, 1972a; Sadlerochit Spring, 1972a, Shublik Spring and Creek, 1972a; Canning River, spring tributary, 1972a, $1973^{\mathrm{a}}$.

Arcynopteryx dichroa (McLachlan, 1872) (=A. compacta McLachlan, 1872)

New RECORDs.-Ribdon River spring, 1m, 1/7/2001; Echooka Spring, 2m \& 3f, 4/7/2001; Lake E5 near Toolik Lake, 1m, 11/7/2001; Toolik Lake, 5f, 21/7/2001; Kuparuk River "lower" 
aufeis, 4m \& 8f, 19/6/2004; Galbraith Lake "campground spring," 10m, 24/6/2007; Ivishak Hot Spring, hillslope source, 6m, 26-29/5/ 2008; Kuparuk River (Dalton Highway bridge), If, 23/6/2012; Kuparuk River "lower" aufeis, 23/6/2012, 3m, lf \& 2 larvae, 10/8/2012; Toolik Lake, 1 brachypterous m, 30/6/2012, 1f, 1/7/ 2012.

Historical RECORDS.-Anaktuvuk River, 1949a,c; Kukpuk River, 1961a; Sadlerochit Spring, 1966 a; Shublik Spring, 1966 ${ }^{\mathrm{a}}$; Echooka River, 1971a, Ribdon spring tributary, 1971 a, Echooka Spring, 1971a, 1972a, Canning River, 1972a; Marsh Fork of Canning River, 1972a; Shublik Spring, 1972a; Canning River, spring tributaries, 1972a, 1973a; Feniak Lake, 1973a; Shrader and Peters Lakes, 1973a; Toolik Lake, 1976a; Atigun River, 1977a; "Oil Spill Lake" near Toolik Lake, 1978a; Toolik Lake, 1979a.

\section{TRICHOPTERA}

Glossosomatidae

*Glossosoma intermedium (Klapálek, 1892)

NEW RECORDS.-Ivishak Hot Spring stream, $1 \mathrm{~m}, 23-28 / 6 / 2002,2 \mathrm{~m}$ \& 2f, 26-29/5/2008; Ivishak Hot Spring, hillslope source, $3 \mathrm{~m}$ \& 10f, $26-29 / 5 / 2008$.

\section{*Glossosoma sp.}

NEW RECORDS.-Sadlerochit Spring, numerous larvae and pupae, 22/7/2002; Kuparuk River (Dalton Highway bridge), larvae, 2/8/ 2012, 27/8/2012.

\section{Hydroptilidae}

**Agraylea cognatella McLachlan, 1880

New Records.-Toolik Lake, $18 \mathrm{~m} \& 4 \mathrm{f}$, 21/7/2001, numerous $m$ \& f, 6/7/2012; Dan Creek, 2m, 30/6/2012.

Notes.-The range of Agraylea cognatella is restricted to the Yukon, Alaska, and Asia (Wiggins and Parker 1997).

\section{*Ochrotrichia logana (Ross, 1941)}

NEW RECords.-Krugurak Spring, Noatak River drainage, many mf metamorphotypes, 18/7/2003.

Notes.-Ruiter (1999) first reported $O$. logana from southern Alaska (Katmai Pennisula). Our Noatak River drainage record establishes the presence of this wide-ranging species (northern Mexico to Alaska; Ruiter 1999, Bueno-Soria 2009), in the North American Arctic.

\section{*Oxyethira sp.}

NEW RECORDS.-Toolik Lake, Lake I-3 outlet stream near Toolik Lake, larvae, 15/7/2005; Lake I-6 Headwater outlet stream near Toolik Lake, larvae, 15/7/2005.

Notes.-Oxyethira araya Ross, 1941 has been recorded from the northern Yukon Territory, Canada (Wiggins and Parker 1997).

$$
\text { Rhyacophilidae }
$$

$$
\text { **Rhyacophila mongolica Schmid, }
$$
Arefina \& Levanidova 1993

NEW RECORDS.-Kuparuk River (Dalton Highway bridge), larvae, 20/7/2001, larvae, 11/7/2001, 22m, 14/7/2001; Oksrukuyik Creek, $1 \mathrm{~m}$ from arctic grayling (Thymallus arcticus) gut content, 8m, 21/7/2003; Oksrukuyik Creek, 1m, 9/6/2011; Hershey Creek, 9m, 15/7/2011, 13m, 9/7/2012, 3m, 27/7/2012; Dan Creek, 25m, 7/7/2012, 6m, 21/7/2012, 2m, 29/7/2012; Happy Valley Creek, 5m, 7/7/2012, 1m, 29/7/ 2012; Kuparuk River (Dalton Highway bridge), 10m, 2/7/2012, 1m, 15/7/2012, 1m, 27/7/2012.

Notes.-Rhyacophila mongolica has been reported from the Yukon (Wiggins and Parker 1997). Prior to that record, this species was only reported for Europe, northeastern Asia, and Mongolia (Chuluunbat and Morse 2007).

Apataniidae

Apatania zonella (Zetterstedt, 1840)

NEW RECORDS.-Galbraith Lake "campground spring," abundant larvae, pupae and adult females, 24/6/2007, abundant larvae, pupae and adult females, 27/6/2008, abundant larvae and pupae, 30/5/2008.

Historical RECORDS.-Anaktuvuk Pass, 1949b,c; Point Barrow, $1949^{\mathrm{b}, \mathrm{c}}$.

*Apatania stigmatella (Zetterstedt, 1840)

New RECORDS.-Lupine Creek, 1m, 11/8/ 2011; Franklin Bluffs at Dalton Highway Milepost $386,1 \mathrm{~m}, 12 / 8 / 2012$.

\section{Apatania. sp.}

New RECORD.-Kuparuk River "upper" aufeis, larva, 8/6/2012. 


\section{Brachycentridae}

Brachycentrus americanus (Banks, 1899)

New RECORDs.-Kuparuk River (Dalton Highway bridge), $5 \mathrm{~m} \& 3 \mathrm{f}, 20 / 7 / 2001$; Sadlerochit Spring, larvae, $5 \mathrm{~m} \&$ \& 1 , 22/7/2002; Dan Creek, 2m, 7/7/2012, 1m, 30/6/2012.

HistORICAL RECORDS.-Atigun River, 1979b; Kuparuk River, 1984-1986h.

\section{\$Micrasema gelidum McLachlan, 1876}

NEW RECORDS.-Kuparuk River (Dalton Highway bridge), $21 \mathrm{~m}$ \& 12f, 20/7/2001; Lake E-1 outlet near Toolik Lake, 1m, 9/6/2011; Kuparuk River (Dalton Highway bridge), 3m, 2/7/2011, 1m, 15/7/2011, 1m, 4/7/2012; Hershey Creek, 2m, 15/7/2011, 3m, 9/7/2012; Dan Creek, 1m, 7/7/2012; Wolf Creek, 1m, 21/7/ 2012.

Historical RECORDS.-Nanushuk River, 1949b,c; Pt. Barrow, 1949b,c (as M. scissum McLachlan); Pt. Barrow, 1975b ${ }^{\mathrm{b}}$ 1979b; Arctic slope of Yukon Territory, Canada (no date) ${ }^{\mathrm{k}}$.

\section{Leptoceridae}

Mystacides sepulchralis (Walker, 1852)

NEW RECORDS.-Toolik Lake, 3m, 12/7/2011; Dan Creek, 1m, 7/7/2012, 2m, 21/7/2012.

HistORICAL RECORDS.- $3.2 \mathrm{~km}$ above mouth of Kuguroruk River, 5/8/1981 ${ }^{\mathrm{b}}$.

\section{Limnephilidae}

Arctopora trimaculata (Zetterstedt, 1840)

Historical RECORD.-Symbol on distribution map (Fig. 19, Wiggins and Parker 1997) indicates a record from the vicinity of the lower Colville River; exact location not reported.

\section{**Asynarchus aldinus (Ross, 1941)}

NEW RECORDS.-Imnavait Creek, 1m, 27/7/ 2011; Happy Valley Creek, 1m, 29/7/2012; Lupine Creek, 1m, 11/8/2012.

NoTE.-Asynarchus aldinus is widely distributed along the Rocky Mountains (Wiggins and Parker 1997) from the foothills of the North Slope of Alaska south to Utah.

\footnotetext{
*\$Asynarchus lapponicus (Zetterstedt, 1840)
}

NEW RECORDS.-Wolf Creek, 3m, 4/8/2011, 1m, 10/8/2011; Kuparuk River (Dalton Highway bridge), $1 \mathrm{~m}, 31 / 7 / 2012$.
Historical RECORDS.-Arctic slope of Yukon Territory, Canada (no date) ${ }^{\mathrm{k}}$.

*Asynarchus montanus (Banks, 1907)

NEW RECORDS.—Dan Creek, 1m, 29/7/2012.

*Dicosmoecus obscuripennis Banks, 1938

New ReCords.-May Creek spring, larvae, 8/7/2001; Kuparuk River (Dalton Highway bridge), 1m, 20/7/2001, 1m, 9/8/2001; Kuparuk River "lower" aufeis, pupal aggregation (photographed), 19/6/2004; Hershey Creek, 2m, 9/7/2012, 1m, 15/7/2012; Kuparuk River (Dalton Highway bridge), 1m, 15/7/2012, 4m, 27/7/2012; Dan Creek, 3m, 21/7/2012; Wolf Creek, 1m, 21/7/2012, 1m, 9/7/2012, 3m, 7-14/7/2012.

\section{Dicosmoecus sp.}

Historical RECORDS.- Jago River, larvae, 1982-1983g.

\section{Ecclisomyia conspersa Banks, 1907}

NEw RECORDS.-Echooka Spring, 1m, 4/7/ 2001; Ivishak River tributary nr. Ivishak Hot Spring, 1m, 23-28/6/2002; Sadlerochit Spring, $1 \mathrm{~m}, 22 / 7 / 2002$; Toolik Lake, 1m, 26/6/2001, 1m, 29/6/2001, 1m, 28/6/2001; Ivishak Hot Spring stream, $1 \mathrm{~m}, 2-6 / 7 / 2007$, $1 \mathrm{~m}, 22 / 4 / 2008$, $1 \mathrm{~m}, 26 / 5 / 2008$; Ivishak River tributary nr. Ivishak Hot Spring, 1m, 28/5/2008; Ivishak River, main stem nr. Ivishak Hot Spring, 1m, 28/5/2008; Ivishak Hot Spring, hillslope source, 1m, 26-29/5/2008; Kuparuk River, "lower" aufeis, $1 \mathrm{~m}, 8 / 6 / 2012$; Cobblestone Spring, $1 \mathrm{~m}$, 8/6/2012; Galbraith Lake, 1m, 15/6/2012.

Historical RECORDS.-Wrench Creek, Noatak River drainage, 4/8/1981 ${ }^{\mathrm{b}}$.

\section{Ecclisomyia sp.}

Historical ReCORds.- Jago River, larvae, 1982-1983g; Canning River, larvae, 19821983g; Carter Creek, larvae, 1982-1983g.

Grammotaulius alascensis Schmid, 1964 (= G. subborealis Schmid, 1964)

New Records.-Percy Pingo, 1m, 31/7/2011.

Historical RECORDS.-Umiat, 31/7/1959e; Jago Lake, 1m, 2/8/1957e.

\section{Grammotaulius sp.}

New Records.-Toolik River at Dalton Highway, 1 larva, 3/7/2001. 
Grensia praeterita (Walker, 1852)

NEW RECORDS.-Toolik Lake, 1m, 28/6/2001; Kuparuk River "upper" aufeis, 1f, 29/6/2007; Barrow Environmental Observatory, $2 \mathrm{~m}$ \& 1f, 18/6/2011; Toolik Lake, 1m, 3/6/2012; Lupine Creek, 2m, 6/6/2012; Galbraith Lake, 3m, 6/6/2012; May Creek aufeis, 2f, 8/6/2012; Kuparuk River (Dalton Highway bridge), 5m, 10/6/2012; Tea Lake, 1m, 26/6/2012; Dalton Highway Mile Post 386, 3m, 4/9/2012.

Historical RECORDS.-Anuktuvuk Pass, 1948,c; Meade River, 1950b; Ogutoruk Creek, Cape Thompson, 1966 b; Inaru River, 28/9/no year $^{b, c}$; Prudhoe Bay, no year ${ }^{b}$.

†Lenarchus expansus Martynov, 1914

Historical RECORDS.-Point Barrow, $1898^{\mathrm{b}}, 1958^{\mathrm{b}}$; Arctic slope of Yukon Territory, Canada (no date) ${ }^{\mathrm{k}}$.

*Hydatophylax variabilis (Martynov, 1910)

NEW RECORDS.-Kuparuk River, "lower" aufeis, 2f, 8/6/2012; May Creek aufeis, 1m, 8/6/2012.

Notes.-Hydatophylax variabilis has been previously reported from Sweden to eastern Siberia in Eurasia, as well in the coastal regions of southeastern Alaska. It is thus the only essentially Palearctic trichopteran species also known to occur in southern Alaska but nowhere else in North America (Nimmo 1986, Wiggins and Parker 1997). Our record from the North Slope is the first to document the presence of $H$. variabilis in Beringia.

*Limnephilus argenteus Banks, 1914

NEW RECORDS.-Oksrukuyik Creek, 1m, 9/6/2011.

**Limnephilus diphyes McLachlan, 1880

New RECORDs.-Kuparuk River (Dalton Highway bridge), 1m, 4/7/2012.

NoTE.-Limnephilus diphyes has previously been reported from Scandinavia eastward through northwestern Siberia to the Yukon Territory, Canada (Wiggins and Parker 1997).

*Limnephilus femoralis Kirby, 1937

NEW RECORDS.-Lake E-1 outlet near Toolik Lake, 1m, 9/6/2011.

*Limnephilus fenestratus (Zetterstedt, 1840)

NEw RECORDs.-Franklin Bluffs near Dal- ton Highway Milepost 386, 1m, 12/8/2012; Dalton Highway Milepost 355, 1m, 12/8/2012.

†Limnephilus kennicotti Banks, 1920

Historical RECORDS.-Arctic slope of Yukon Territory, Canada (no date) $)^{\mathrm{k}}$.

*Limnephilus nigriceps (Zetterstedt, 1840)

New RECORDs.-Dan Creek, 2m, 23/8/ 2012; Kuparuk River (Dalton Highway bridge), $1 \mathrm{~m}, 24 / 8 / 2012$.

\section{+Limnephilus pallens Banks, 1920}

Historical RECORDS.-Arctic slope of Yukon Territory, Canada (no date) $)^{\mathrm{k}}$.

* \$imnephilus picturatus McLachlan, 1875

NEW RECORDS.-Franklin Bluffs near Dalton Highway Milepost 386, many males, 22/7/2012.

Historical RECORDs.-Arctic slope of Yukon Territory, Canada (no date) ${ }^{\mathrm{k}}$.

*Limnephilus sansoni Banks, 1918

New RECORDS.-Kuparuk River (Dalton Highway bridge), If, 24/8/2012; Mosquito Lake, 1m, 1/8/2012.

Onocosmoecus unicolor (Banks, 1897)

New RECORDs.-Sadlerochit Spring, numerous larvae and adults, 22/7/2002.

Historical RECORDS.-Wrench Creek, Noatak River drainage, 4/8/1981 b; Noatak River near confluence with Kuguroruk River, 31/7/1981 ; Sadlerochit Spring, no yeard.

Molannidae

*Molannodes tinctus (Zetterstedt, 1840)

NEW RECORDS.-Dan Creek, 1m, 29/7/2012.

Phryganeidae

¥Agrypnia obsoleta (Hagen, 1864)

Historical RECORDs.-Arctic slope of Yukon Territory, Canada (no date) ${ }^{\mathrm{k}}$.

\$Agrypnia pagetana Curtis, 1835

Historical RECORDS.-Noatak River, $1 \mathrm{~m}$ \& 1f, 25/6/1973f; Arctic slope of Yukon Territory, Canada (no date) ${ }^{\mathrm{k}}$.

Agrypnia sahlbergi (McLachlan, 1880)

Historical RECORD.-Noatak River, $1 \mathrm{~m}$, 2/8/1973f. 
Agrypnia sp.

New Records.-Percy Pingo, 1 larva, $31 / 7 / 2011$.

Banksiola crotchi Banks, 1944

Historical RECORDS.-Noatak River near confluence with Charley River, 1f, 14/6/1974f.

\section{Banksiola sp.}

HistORICAL RECORDS.-Point Barrow 1949b,c.

Oligotricha lapponica (Hagen, 1864)

New Records.-Oil Spill Hill near Dalton Highway, lf, 28/6/2007; Kuparuk River "upper" aufeis, lf, 29/6/2007.

Historical RECORDS.—Pt. Barrow, 1f, 29/7/ $1949^{f}$.

\section{Discussion}

Previous studies have summarized reported occurrences of Trichoptera and Plecoptera from the entire state of Alaska (Nimmo 1986, Stewart and Oswood 2006). Here we update these lists for the North Slope region of Alaska with 2 new species records of Plecoptera and 19 new records of Trichoptera (4 new state records). New state records for Trichoptera include Agraylea cognatella, Rhyacophila mongolica, Asynarchus aldinus, and Limnephilus diphyes. New records of Trichoptera for the North Slope include Glossosoma intermedium, Ochrotrichia logana, Oxyethira sp., Apatania stigmatella, Asynarchus lapponicus, A. montanus, Dicosmoecus obscuripennis, Hydatophylax variabilis, Limnephilus argenteus, L. femoralis, L. fenestratus, L. nigriceps, L. picturatus, L. sansoni, and Molannodes tinctus. New records for Plecoptera on the North Slope include Isocapnia cf. integra and Paraperla frontalis.

Our species list for the North Slope contains a total of 24 and 33 species of Plecoptera and Trichoptera, respectively. If records from the physiographically equivalent area of the Yukon Territory are included (Wiggins and Parker 1997), the total richness of this region is 25 species of stoneflies and 36 species of caddisflies. Compared to the "north slope" of the Yukon, which includes the northward flowing drainages of the Arctic Coastal Plain, the British Mountains, and the Arctic Plateau (Wiggins and Parker 1997), the North Slope region of Alaska has a substantially higher species richness of these groups. Alaska North Slope richness is 24 for Plecoptera and 33 for Trichoptera, compared to richness on the Yukon "north slope," which is 3 for Plecoptera and 8 for Trichoptera. Because a significant investment in biotic inventories has been made in the Yukon Territory (e.g., Wiggins and Parker 1997, Stewart and Ricker 1997), we attribute this difference to factors such as greater habitat diversity due to a much greater geographical area on Alaska's North Slope rather than to differences in collecting effort in the different regions.

Although taxonomic inventories will almost always be incomplete, our assessment of the stoneflies and caddisflies seems sufficient to support 2 major conclusions about factors underlying the level of biodiversity documented. First, the role of Beringia (i.e., the region encompassing eastern Siberia, the Bering land bridge, and most of present-day Alaska and the Yukon Territory during much of the Pleistocene) as a biogeographic refuge during the Pleistocene has had a significant effect on the taxonomic structure of the freshwater insects of the North Slope. There has been much interest in the role of Beringia in determining patterns of Arctic biodiversity (e.g., Wiggins and Parker 1997, Stewart and Ricker 1997). Because this region remained largely ice-free during the Pleistocene, it functioned as a refuge for taxa that were extirpated by continental glaciers elsewhere. Caddisfly species showing such distributions include Agraylea cognatella, Rhyacophila mongolica, Arctopora trimaculata, Dicosmoecus obscuripennis, Grammotaulius alascensis, Grensia praeterita, Hydatophylax variabilis, Lenarchus expansus, Limnephilus diphyes, L. fenestratus, Agrypnia obsoleta, A. sahlbergi, and Oligotrichia lapponica (36\%). Species of Plecoptera showing ranges that are either Beringian or PalearcticBeringian include Mesocapnia variabilis, $\mathrm{Ne}$ moura normani, Podmosta weberi, and Alaskaperla ovibovis (17\%).

Although the contribution of Beringian taxa to the plecopteran fauna of the North Slope is modest, the contribution of such taxa to the trichopteran fauna is substantial. These results can be compared with species lists from Churchill Manitoba, Canada, where 66 species of caddisfly and 16 species of stonefly were found (Zhou et al. 2010). Of the 66 caddisflies found at Churchill, only 17 of the same species 
are also found in our list. Of the 16 species of stoneflies found at Churchill, only 3 species are also found in our list. The structure of the trichopteran species diversity of the North Slope therefore represents a little known but relatively unique biogeographic heritage that should be of clear conservation concern given the rapidly changing climate of the Arctic (ACIA 2005).

The second major conclusion about factors underlying the level of plecopteran and trichopteran biodiversity of the North Slope is the critical effect of freshwater habitats associated with perennial springs. A number of spring streams found along the northern foothills of the Brooks Range flow year-round due to winter water temperatures ranging from 4 to $11{ }^{\circ} \mathrm{C}$, whereas other freshwater habitats with depths $<1.5 \mathrm{~m}$ freeze solid. Although perennial spring streams provide a negligible amount of habitat ( $<1 \%$ of total stream length) on the North Slope, they have important consequences for biodiversity because they provide $100 \%$ of flowing stream habitat during winter (Huryn et al. 2005, Huryn and Hobbie 2012). This habitat is critical for stream organisms that occur on the North Slope but which are unable to tolerate freezing, including a number of freshwater insects, the Dolly Varden char (Salvelinus malma), and the American Dipper (Cinclus mexicanus), a semiaquatic songbird. Another important habitat related to the presence of springs is aufeis. An aufeis is a mass of ice formed by the successive freezing of overlying sheets of water that flow from a spring. The volume of an aufeis can be enormous, ranging up to $5+$ meters thick and many square kilometers in area. Sediments underlying an aufeis remain saturated with unfrozen water year-round and, like the spring streams, provide significant habitat for aquatic insects. Stonefly species that are closely associated with aufeis fields where their larvae inhabit interstitial habitats (e.g., Stanford and Gaufin 1974) include Capnia confusa, C. nearctica, Isocapnia crinita, I. grandis, I. cf. integra, Plumiperla diversa, Suwallia autumna, S. starki, and Paraperla frontalis. Stonefly species that are primarily found in association with spring streams include Podmosta weberi, Zapada columbiana, Z. haysi, T. pacificum, Isoperla decolorata, I. petersoni, and Arcynopteryx dichroa. Although no trichopteran species are specifically associated with aufeis fields, Glos- sosoma intermedium, Ochrotrichia logana, Hydatophylax variabilis, and Oncosmoecus unicolor have been found only in perennial spring habitats on the North Slope. Although comprising $<1 \%$ of the total stream length of the North Slope, perennial spring and aufeis habitats contain about $70 \%$ of the known stonefly species and 10\% of the known caddisfly species.

The 2 major factors contributing to the unique aquatic insect communities of Alaska's Arctic North Slope include the North Slope as a Beringian refuge from continental ice sheets and the occurrence of spring streams as refuge from seasonal winter ice. The North Slope of Alaska is predicted to undergo changes in climate and extent of industrialization in the coming years. This species list, which represents numerous new records for Alaska's North Slope, will aid in our understanding of how aquatic insect communities may respond to these predicted, yet unknown, changes on Alaska’s North Slope.

\section{ACKNOWLEDGMENTS}

We thank Stephanie Parker, Ashley Asmus, Heidi Rantala, Eve Kendrick, Heidi Golden, and Chelsea Smith for collecting specimens for this study. Thanks also to Richard Baumann for taxonomic help with Isocapnia cf. integra and for comments on the species lists. We thank Mac Butler for assistance at Barrow Environmental Observatory. Eve Kendrick, Julia LaRouche, Pat Tobin, and Lauren Koenig assisted with sticky traps. Collections from North Slope habitats from 2001 to 2012 were made possible by several grants from the National Science Foundation (DEB 9810222, ARC 9911278, ARC 0611995, and ARC 0902126).

\section{Literature Cited}

ACIA. 2005. Arctic climate impact assessment. Cambridge University Press. 1042 pp.

Bueno-Soria, J. 2009. A review of the genus Ochrotrichia Mosely (Trichoptera: Hydroptilidae) from Mexico and Central America. Transactions of the American Entomological Society 135:59-160.

Chapin, F.S., III, M. Hoel, S.R. Carpenter, J. LubChenco, B. Walker, T.V. Callaghan, C. Folke, S.A. Levin, K.-G. Mäler, C. Nilsson, et AL. 2006. Building resilience and adaptation to manage Arctic change. AMBIO: A Journal of the Human Environment 35:198-202. 
Chen, I., J.K. Hill, R. Ohlemüller, D.B. Roy, And C.D. THOMAs. 2011. Rapid range shifts of species associated with high levels of climate warming. Science 333(6045):1024-1026.

Chuluunbat, S., and J.C. Morse. 2007. Caddisflies (Insecta: Trichoptera) of Selenge River Basin, Mongolia. Pages 51-57 in J. Bueno-Soria et al., editors, Proceedings of the XIIth International Symposium on Trichoptera, June 18-22, 2006. The Caddis Press, Columbus, OH. xii +378 pp.

de Rham, L.P., T.D. Prowse, and B.R. Bonsal. 2008 Temporal variations in river-ice break-up over the Mackenzie River basins, Canada. Journal of Hydrology 349:441-454.

Glesne, R.S., and S.J. Deschermeier. 1984. Abundance, distribution and diversity of aquatic macroinvertebrates on the North Slope of the Arctic National Wildlife Refuge, 1982 and 1983. Fairbanks Fishery Resources Progress Report 84/2, February 2, 1984. Fishery Resources, U.S. Fish and Wildlife Service.

Hershey, A.E., W.B. Bowden, L.A. Deegan, J.E. HobBie, B.J. Peterson, G.W. Kipphut, G.W. Kling, M.A. Lock, R.W. MerRitT, AND M.C. Miller. 1997. The Kuparuk River: a long-term study of biological and chemical processes in an Arctic river. Pages 107-129 in A.M. Milner and M.W. Oswood, editors, Freshwaters of Alaska. Springer-Verlag, New York, NY. 369 pp.

Hinzman, L.D., N.D. Bettez, W.R. Bolton, F.S. Chapin, M.B. Dyurgerov, C.L. Fastie, B. Griffith, R.D. Hollister, A. Hope, H.P. Huntington, et AL. 2005. Evidence and implications of recent climate change in northern Alaska and other Arctic regions. Climatic Change 72:251-298.

Huryn, A., AND J. Hobbie. 2012. Land of extremes: a natural history of the Arctic North Slope of Alaska. University of Alaska Press, Fairbanks, AK. 311 pp.

Huryn, A.D., K.A. Slavik, R.L. Lowe, S.M. Parker, D.S. Anderson, and B.J. Peterson. 2005. Landscape heterogeneity and the biodiversity of Arctic stream communities: a habitat template analysis. Canadian Journal of Fisheries and Aquatic Sciences 62: 1905-1919.

Martin, P.D., J.L. Jenkins, F.J. Adams, M.T. Jorgenson, A.C. Matz, D.C. Payer, P.E. Reynolds, A.C. TidWELL, AND J.R. ZELENAK. 2009. Wildlife response to environmental Arctic change. U.S. Fish and Wildlife Service, Fairbanks, AK.

MCCAFFERTY, W.P. 1985. The Ephemeroptera of Alaska. Proceedings of the Entomological Society of Washington 87:381-386.

Nimmo, A.P. 1986. Preliminary annotated checklist of the Trichoptera (insects) of Alaska. Contributions to Natural Science, British Columbia Provincial Museum $5: 1-7$.

RANDOLPH, R.P., AND W.P. MCCAFFERTy. 2005. The mayflies (Ephemeroptera) of Alaska, including a new species of Heptageniidae. Proceedings of the Entomological Society of Washington 107:190-199.

Ruiter, D.E. 1999. A new species and new synonym in the genus Psychoronia (Limnephilidae), with significant records for caddisflies (Trichoptera) from west- ern North America. Great Basin Naturalist 59: $160-168$.

Schmid, F. 1964. Some Nearctic species of Grammotaulius Kolenati (Trichoptera: Limnephilidae). Canadian Entomologist 96:914-917.

Stanford, J.A., And A.R. Gaufin. 1974. Hyporheic communities of two Montana rivers. Science 185(4152): 700-702.

STEWART, K.W., AND M.W. Oswood. 2006. The stoneflies (Plecoptera) of Alaska and Western Canada. Caddis Press, Columbus, OH.

Stewart, K.W., and W.E. Ricker. 1997. Stoneflies (Plecoptera) of the Yukon. Pages 201-222 in H.V. Danks and J.A. Downes, editors, Insects of the Yukon. Biological Survey of Canada (Terrestrial Arthropods), Ottawa, Ontario, Canada.

Tape, K.D., D. Verbyla, and J. Welker. 2011. Twentieth century erosion in Arctic Alaska: the influence of shrubs, runoff, and permafrost. Journal of Geophysical Research, Biogeosciences 116(G4):G04024

Weber, N.A. 1950. A survey of the insects and related arthropods of Arctic Alaska. Part 1. Transactions of the American Entomological Society (1890- ) 76: 147-206.

White, K.D., A.M. Tuthill, C.M. Vuyovich, and P.B. WEYRICK. 2007. Observed climate variability impacts and river ice in the United States. Proceedings, 14th Workshop on the Hydraulics of Ice Covered Rivers, Quebec City, Canada, June 20-22, 2007. CD format, Paper No. a24: [no page numbers]. CGU-HS Committee on River Ice Processes and the Environment, Edmonton, Alberta, Canada.

WigGins, G.B. 1996. Larvae of the North American caddisfly genera. 2nd edition. University of Toronto Press, Toronto, Ontario, Canada.

1998. The caddisfly family Phryganeidae (Trichoptera). University of Toronto Press, Toronto, Ontario, Canada.

Wiggins, G.B., and C.R. Parker. 1997. Caddisflies (Trichoptera) of the Yukon, with analysis of the Beringian and Holarctic species of North America. Pages 787-866 in H.V. Danks and J.A. Downes, editors, Insects of the Yukon. Biological Survey of Canada (Terrestrial Arthropods), Ottawa, Ontario, Canada.

Wiggins, G.B., AND J.S. Richardson. 1986. Revision of the Onocosmoecus unicolor group (Trichoptera: Limnephilidae, Dicosmoecinae). Psyche 93:187-216.

Zenger, J.T., and R.W. Baumann. 2004. The Holarctic winter stonefly genus Isocapnia, with an emphasis on the North American fauna (Plecoptera: Capniidae). Monographs of the Western North American Naturalist 2:65-95.

Zhou, X., L.M. Jacobus, R.E. Dewalt, S.J. Adamowicz, AND P.D. Hebert. 2010. Ephemeroptera, Plecoptera, and Trichoptera fauna of Churchill (Manitoba, Canada): insights into biodiversity patterns from DNA barcoding. Journal of the North American Benthological Society 29:814-837.

Received 10 August 2013 Accepted 2 July 2014 